\title{
Three-boson bound states in finite volume with EFT
}

\author{
S. Kreuzer ${ }^{1, a}$ and H.-W. Hammer ${ }^{1}$ \\ Helmholtz-Institut für Strahlen- und Kernphysik and Bethe Center for Theoretical Physics, \\ Universität Bonn, 53115 Bonn, Germany
}

\begin{abstract}
The universal properties of a three-boson system with large scattering length are well understood within the framework of Effective Field Theory. They include a geometric spectrum of shallow three-body bound states called "Efimov states" and log-periodic dependence of scattering observables on the scattering length. We investigate the modification of this spectrum in a finite cubic box using a partial wave expansion. The dependence of the binding energies on the box size is calculated for systems with positive and negative two-body scattering length. We compare the full results to results obtained using an expansion around the infinite volume binding energy. The renormalization of the Effective Field Theory in the finite volume is verified explicitly.
\end{abstract}

\section{Introduction}

Few-body systems with resonant interactions characterized by a large scattering length $a$ show interesting universal properties. If $a$ is positive, two particles of mass $m$ form a shallow dimer with energy $E_{2} \approx-\hbar^{2} /\left(m a^{2}\right)$, independent of the mechanism responsible for the large scattering length. Examples for such shallow dimer states are the deuteron in nuclear physics, the ${ }^{4} \mathrm{He}$ dimer in atomic physics, and possibly the new charmonium state $X(3872)$ in particle physics $[1,2]$. In the three-body system, the universal properties include the Efimov effect [3]. If at least two of the three pairs of particles have a large scattering length $|a|$ compared to the range $r_{0}$ of their interaction, there is a sequence of three-body bound states with energies spaced geometrically between $-\hbar^{2} / m r_{0}^{2}$ and $-\hbar^{2} / m a^{2}$. In the limit $1 / a \rightarrow 0$, there are infinitely many bound states with an accumulation point at the three-body scattering threshold. These Efimov states or trimers have a geometric spectrum [3]:

$$
E_{3}^{(n)}=-\left(e^{-2 \pi / s_{0}}\right)^{n-n_{*}} \hbar^{2} \kappa_{*}^{2} / m,
$$

which is specified by the binding momentum $\kappa_{*}$ of the Efimov trimer labeled by $n_{*}$. This spectrum is a consequence of a discrete scaling symmetry with discrete scaling factor $e^{\pi / s_{0}}$. In the case of identical bosons, $s_{0} \approx 1.00624$ and the discrete scaling factor is $e^{\pi / s_{0}} \approx 22.7$. The discrete scale invariance persists if $a$ is large but finite, but in this case it connects states corresponding to different values of the scattering length. The scaling symmetry becomes also manifest in the log-periodic dependence of scattering observables on the scattering length $a$ [4]. The consequences of discrete scale invariance and "Efimov physics" can be calculated in an effective field theory for short-range interactions, where the Efimov effect appears as a consequence of a renormalization group limit cycle [5].

\footnotetext{
${ }^{a}$ e-mail: kreuzer@hiskp.uni-bonn.de
}

While the Efimov effect was established theoretically already in 1970, first experimental evidence for an Efimov trimer in ultracold Cs atoms was provided only recently by its signature in the three-body recombination rate [6]. It could be unravelled by varying the scattering length $a$ over several orders of magnitude using a Feshbach resonance. Since this pioneering experiment, there was significant experimental progress in observing Efimov physics in ultracold quantum gases. More recently, evidence for Efimov trimers was also obtained in atom-dimer scattering [7] and in three-body recombination in a balanced mixture of atoms in three different hyperfine states of ${ }^{6} \mathrm{Li}[8,9]$, in a mixture of Potassium and Rubidium atoms [10], and in an ultracold gas of ${ }^{7} \mathrm{Li}$ atoms [11]. In another experiment with Potassium atoms [12], two bound trimers were observed.

The observation of Efimov physics in nuclear and particle physics systems is complicated by the inability to vary the scattering length and one has to focus on the detection of excited states. While two-neutron halo nuclei could be bound due to the Efimov effect, the analysis of known halo nuclei does not show much promise for an unambiguous identification (See Ref. [13] and references therein). Another opportunity to observe Efimov physics is given by lattice QCD simulations of three-nucleon systems [14]. A number of studies of the quark-mass dependence of the chiral nucleon-nucleon $(N N)$ interaction found that the inverse scattering lengths in the relevant ${ }^{3} S_{1}-{ }^{3} D_{1}$ and ${ }^{1} S_{0}$ channels may both vanish if one extrapolates away from the physical values to slightly larger quark masses [1517]. This implies that QCD is close to the critical trajectory for an infrared renormalization group limit cycle in the three-nucleon sector. It was conjectured that QCD could be tuned to lie precisely on the critical trajectory by tuning the up and down quark masses separately [18]. As a consequence, the triton would display the Efimov effect. More refined studies of the signature of Efimov physics in this case followed $[19,20]$. However, a proof of this conjecture 
can only be given by an observation of this effect in a lattice QCD simulation [14]. The first full lattice QCD calculation of nucleon-nucleon scattering was reported in [21] but statistical noise presented a serious challenge. A promising recent high-statistics study of three-baryon systems presented also initial results for a system with the quantum numbers of the triton such that lattice QCD calculations of three-nucleon systems are now within sight [22]. For a review of these activities, see Ref. [23]. Since lattice simulations are carried out in a cubic box, it is important to understand the properties of Efimov states in the box. The first step towards this goal is to understand these modifications for a system of three identical bosons.

The corresponding modifications of the Efimov spectrum can be calculated in effective field theory (EFT) since the finite volume modifies the infrared properties of the system. The properties of two-body systems with large scattering length in a cubic box were calculated in Ref. [24]. Some properties of three-body systems in a finite volume have also been studied previously. For repulsive and weakly attractive interactions without bound states, Tan has determined the volume dependence of the ground state energy of three bosons up to $O\left((a / L)^{7}\right)$ [25]. In Refs. [26,27], this result was extended for general systems of $N$ bosons. It was used to analyze recent results for three and more boson systems from lattice QCD [28-30]. For the unitary limit of infinite scattering length, some studies have been carried out as well. The properties of three spin- $1 / 2$ fermions in a box were investigated in [31]. However, this system has no three-body bound states in the infinite volume. The volume dependence of energy levels in a finite volume can also be used to extract scattering phase shifts and resonance properties from lattice calculations $[32,33]$. For a recent application of this idea to the $\Delta(1232)$ resonance, see Refs. [34, 35].

\section{Theoretical Framework}

Our study is carried out to leading order in the large scattering length. This corresponds to the zero range limit with $r_{0}=0$. Our results will be applicable to physical states whose size is large compared to $r_{0}$. Using units with $\hbar=$ $m=1$ from now on, the effective Lagrangian can be written as [1]

$$
\begin{aligned}
\mathcal{L}=\psi^{\dagger} & \left(i \partial_{t}+\frac{1}{2} \nabla^{2}\right) \psi+\frac{g_{2}}{4} d^{\dagger} d \\
& -\frac{g_{2}}{4}\left(d^{\dagger} \psi^{2}+\text { h.c. }\right)-\frac{g_{3}}{36} d^{\dagger} d \psi^{\dagger} \psi+\ldots,
\end{aligned}
$$

where the dots indicate higher order terms. It involves the boson field $\psi$ and an auxiliary dimer field $d$. The coupling constants $g_{2}$ and $g_{3}$ are matched to the scattering length and a three-body observable, respectively.

The atom-dimer amplitude is determined by the inhomogeneous integral equation depicted in Fig. 1, where the single lines denote the boson propagator and the double lines denote the full dimer propagator. In a cubic box, the momenta are quantized and the loop integrals in the equation are replaced by discrete sums over quantized momenta

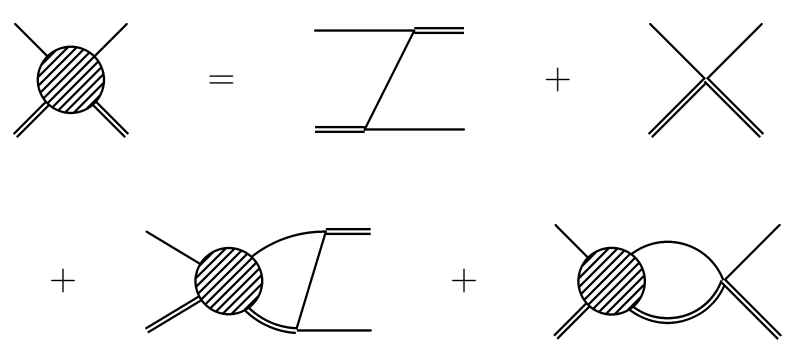

Fig. 1. Integral equation for the atom-dimer amplitude. The single lines denote the boson propagator and the double lines denote the full dimer propagator.

$\mathbf{k}=(2 \pi / L) \mathbf{n}, \mathbf{n} \in \mathbb{Z}^{3}$, where $L$ is the side length of the box. The loop sums are regulated via a momentum cutoff $\Lambda$ just as in the infinite volume case. All observables are independent of $\Lambda$. This is achieved by an appropriate dependence of the coupling constant $g_{3}$ on $\Lambda$ [5]. This dependence can be calculated analytically, but one three-body input is required to fix the unknown constant $\Lambda_{*}$ (see below). This renormalization procedure is performed in the infinite volume limit. The ultraviolet physics associated with the renormalization of the EFT should not interfere with the infrared physics brought about by putting the system into a finite volume, as long as the corresponding scales are well separated. We will verify this expectation explicitly below.

The full dimer propagator $D$ denoted by the double lines in Fig. 1 is obtained by dressing the bare dimer propagator which is simply a constant, $4 i / g_{2}$, with bosonic loops. This leads to an infinite sum which can be evaluated analytically yielding

$$
D(E, \mathbf{0})=\frac{32 \pi}{g_{2}^{2}}\left[\frac{1}{a}-\sqrt{-E}+\frac{1}{L} \sum_{\substack{j \in \mathbb{Z}^{3} \\ \jmath \neq 0}} \frac{1}{|J|} \mathrm{e}^{-|ر| \sqrt{-E} L}\right]^{-1}
$$

for a dimer at rest. The term containing the box length $L$ vanishes in the limit $L \rightarrow \infty$ and the expression reduces to the infinite volume result. Using the Feynman rules encoded in Eq. (2) and the full dimer propagator from above, we can translate the Feynman diagrams in Fig. 1 into an equation for the atom-dimer amplitude. It involves an integration over the loop energy and a sum over the quantized loop momenta. The integration over the loop energy is performed using the residue theorem while the remaining sum over the quantized momenta is rewritten into an integral by virtue of Poisson's resummation formula: $\sum_{\mathbf{n} \in \mathbb{Z}^{3}} \delta^{3}(\mathbf{z}-\mathbf{n})=\sum_{\mathbf{m} \in \mathbb{Z}^{3}} \exp (i 2 \pi \mathbf{m} \cdot \mathbf{z})$, which is understood to be used under an integral.

The resulting expression is simplified further by exploiting the behavior of the atom-dimer amplitude near a bound state. For energies close to a bound state energy, the amplitude has a simple pole, and the dependence on the incoming and outgoing momenta separates. Thus, we obtain a homogeneous integral equation for the bound state amplitude $\mathcal{F}$, namely

$$
\mathcal{F}(\mathbf{p})=\frac{1}{\pi^{2}} \int^{\Lambda} \mathrm{d}^{3} y \sum_{\mathbf{m} \in \mathbb{Z}^{3}} \mathrm{e}^{i L \mathbf{m} \cdot \mathbf{y}} \mathcal{Z}_{E}(\mathbf{p}, \mathbf{y}) \tau_{E}(y) \mathcal{F}(\mathbf{y}),
$$


where

$$
\begin{aligned}
& \mathcal{Z}_{E}(\mathbf{p}, \mathbf{y})=\left(p^{2}+\mathbf{p} \cdot \mathbf{y}+y^{2}-E\right)^{-1}+\frac{H(\Lambda)}{\Lambda^{2}}, \\
& \tau_{E}(y)=\left(-\frac{1}{a}+\sqrt{\frac{3}{4} y^{2}-E}-\sum_{\substack{\mathbf{j} \in \mathbb{Z}^{3} \\
\mathbf{j} \neq \mathbf{0}}} \frac{1}{L|\mathbf{j}|} \mathrm{e}^{-L|\mathbf{j}| \sqrt{\frac{3}{4} y^{2}-E}}\right)^{-1} .
\end{aligned}
$$

The $\Lambda$-dependent three-body interaction in Eq. (5) is given by

$$
H(\Lambda) \equiv-g_{3} \Lambda^{2} /\left(9 g_{2}^{2}\right)=\frac{\cos \left[s_{0} \ln \left(\Lambda / \Lambda_{*}\right)+\arctan s_{0}\right]}{\cos \left[s_{0} \ln \left(\Lambda / \Lambda_{*}\right)-\arctan s_{0}\right]}
$$

where $s_{0} \approx 1.00624$ is a transcendental number [5].

In the infinite volume case, only S-wave bound states are formed. In a cubic box, however, the extraction of the $\mathrm{S}$-wave part of $\mathcal{F}$ is not straightforward due to the breakdown of the spherical symmetry to a cubic symmetry. The infinitely many irreducible representations of the spherical symmetry are mapped onto the five irreducible representations of the cubic symmetry. To investigate the mixing of the different partial waves, the part of the amplitude with quantum number $\ell$ is projected out

$$
\begin{aligned}
F_{\ell}(p)=\frac{4}{\pi} & \int_{0}^{\Lambda} \mathrm{d} y y^{2}\left[Z_{E}^{(\ell)}(p, y) \tau_{E}(y) \frac{1}{2 \ell+1} F_{\ell}(y)\right. \\
& +2 \sqrt{\pi} \sum_{\substack{\mathbf{n} \in \mathbb{Z}^{3} \\
\mathbf{n} \neq 0}} \sum_{\ell^{\prime}, m^{\prime}}^{\left.A_{1}\right)} \sum_{\ell^{\prime \prime}, m^{\prime \prime}}\left(\begin{array}{ccc}
\ell^{\prime} & \ell^{\prime \prime} & \ell \\
0 & 0 & 0
\end{array}\right)\left(\begin{array}{ccc}
\ell^{\prime} & \ell^{\prime \prime} & \ell \\
m^{\prime} & m^{\prime \prime} & 0
\end{array}\right) \\
& \times i^{\ell^{\prime \prime}} \frac{C_{\ell^{\prime} m^{\prime}}}{C_{\ell 0}} Y_{\ell^{\prime \prime} m^{\prime \prime}}(\hat{n}) \sqrt{\frac{\left(2 \ell^{\prime}+1\right)\left(2 \ell^{\prime \prime}+1\right)}{2 \ell+1}} \\
& \left.\times j_{\ell^{\prime \prime}}(L|\mathbf{n}| y) Z_{E}^{(\ell)}(p, y) \tau_{E}(y) F_{\ell^{\prime}}(y)\right] .
\end{aligned}
$$

where we have used Wigner 3- $j$ symbols and

$$
\frac{Z_{E}^{(\ell)}(p, y)}{2 \ell+1}=\left[\frac{1}{p y} Q_{\ell}\left(\frac{p^{2}+y^{2}-E}{p y}\right)+\frac{H(\Lambda)}{\Lambda^{2}} \delta_{\ell 0}\right]
$$

with $Q_{\ell}$ a Legendre function of the second kind. Furthermore, $j_{\ell^{\prime \prime}}$ is a spherical Bessel function of order $\ell^{\prime \prime}$. The $\ell^{\prime \prime}$ sum runs over all partial waves while the $\ell^{\prime}$ sum runs over partial waves associated with the $A_{1}$ representation of the cubic group, namely $\ell^{\prime}=0,4,6, \ldots$ The size of the contribution of the respective partial wave to the cubic group harmonic of the $A_{1}$ representation is given by the coefficients $c_{\ell m}$ (See, e.g., Refs. [36,37]). The first term in Eq. (6) reproduces the equation for the corresponding partial wave of the infinite volume amplitude, while the second term yields corrections due to the quantization of the momenta and admixtures of other partial waves.
For our study of the trimer states in the cubic box, this equation is now specialized to the $\ell=0$ case:

$$
\begin{aligned}
& F_{0}(p)=\frac{4}{\pi} \int_{0}^{\Lambda} \mathrm{d} y y^{2}\left[Z_{E}^{(0)}(p, y) \tau_{E}(y)\right. \\
& \times\left(1+\sum_{\substack{\mathbf{n} \in \mathbb{Z}^{3} \\
\mathbf{n} \neq 0}} \frac{\sin (L|\mathbf{n}| y)}{L|\mathbf{n}| y}\right) F_{0}(y) \\
&+2 \sqrt{\pi} \sum_{\substack{\mathbf{n} \in \mathbb{Z}^{3} \\
\mathbf{n} \neq 0}} \sum_{\ell^{\prime}=4,6, \ldots}^{\left(A_{1}\right)} \sum_{m^{\prime}=-\ell^{\prime}}^{\ell^{\prime}} i^{\ell^{\prime}} j_{\ell^{\prime}}(L|\mathbf{n}| y) Y_{\ell^{\prime} m^{\prime}}^{*}(\hat{n}) \\
&\left.\times Z_{E}^{(0)}(p, y) \tau_{E}(y) C_{\ell^{\prime} m^{\prime}} F_{\ell^{\prime}}(y)\right] .
\end{aligned}
$$

Since the leading term in the expansion of the Bessel functions in Eq. (6) is $1 /(L|\mathbf{n}| y)$ these contributions are suppressed by at least $a / L$. They will be small for volumes not too small compared to the size of the bound state. The lowest partial wave that is mixed with the $\mathrm{S}$-wave is the $\ell=4$ wave. Moreover, contributions from higher partial waves will be suppressed kinematically for shallow states with small binding momentum. This is ensured by the spherical harmonic in the second term of Eq. (6). Only for small lattices, i. e. when $a / L$ is large, this behavior is counteracted by terms stemming from the spherical Bessel function $j_{\ell}(L|\mathbf{n}| y)$ and higher partial waves may contribute significantly. We will therefore mostly neglect the inhomogeneous terms in Eq. (8). For two specific examples, we will show results including the next partial wave $\ell=4$.

\section{Results}

By employing the formalism laid out in the previous section, we have calculated energy levels in finite cubic volumes of varying side lengths. The results of these calculations are presented in the following. For convenience, the dependence of the energies on the boson mass $m$ is reinstated in this section.

\subsection{Positive scattering length}

First, we present results for systems with $a>0$. In this regime, a physical diboson state with binding energy $E_{D}=$ $-1 /\left(m a^{2}\right)$ exists. This energy is therefore identical with the threshold for the break-up of a trimer into a diboson and a single boson. We choose states with different energies in the infinite volume, including shallow as well as deeply bound states:

$$
\begin{array}{cll}
\text { Ia: } & E_{3}^{\infty}=-1.18907 /\left(m a^{2}\right), & \Lambda_{*} a=5.66, \\
\text { Ib: } & E_{3}^{\infty}=-27.4427 /\left(m a^{2}\right), & \Lambda_{*} a=5.66, \\
\text { Ic: } & E_{3}^{\infty}=-9440.91 /\left(m a^{2}\right), & \Lambda_{*} a=5.66, \\
\text { II: } & E_{3}^{\infty}=-5.04626 /\left(m a^{2}\right), & \Lambda_{*} a=1.66, \\
\text { III: } & E_{3}^{\infty}=-11.1322 /\left(m a^{2}\right), & \Lambda_{*} a=3.66 .
\end{array}
$$

Here, $E_{3}^{\infty}$ is the trimer energy in the infinite volume. Note that the states Ia, Ib and Ic appear in the same physical system characterized by $\Lambda_{*} a=5.66$. For each of these states, 

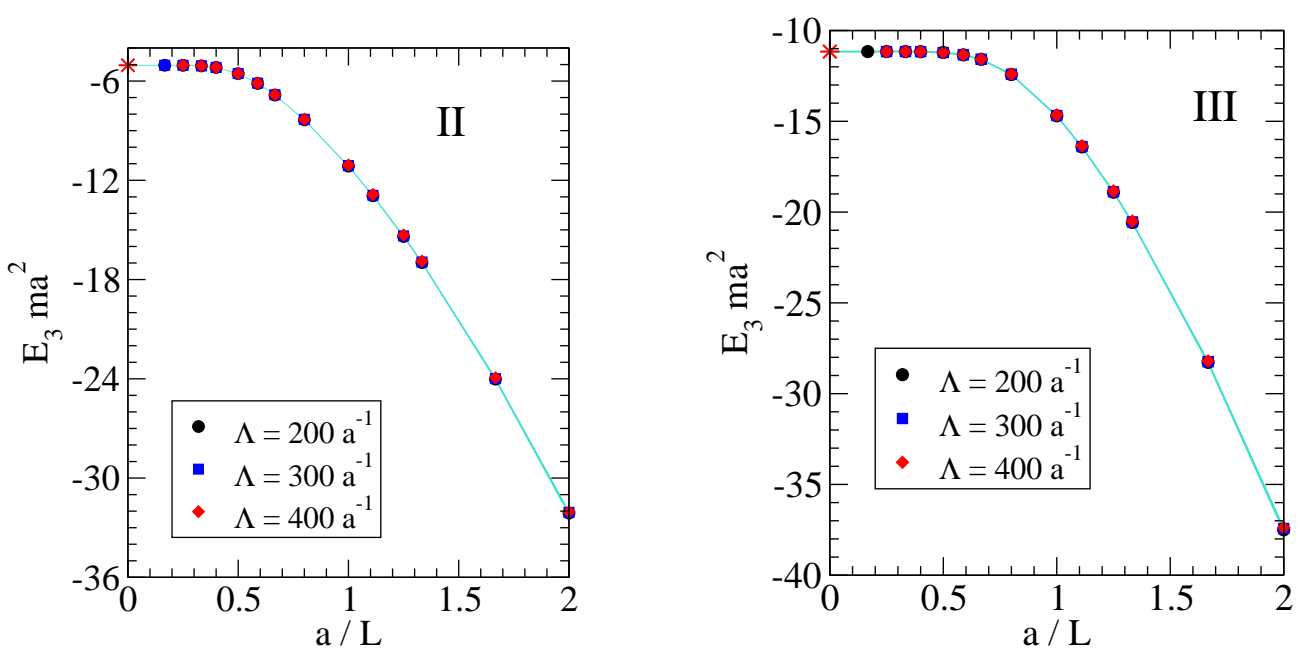

Fig. 2. Variation of the trimer energy $E_{3}$ with the side length $L$ of the cubic volume for the states II (left) and III (right). Plotted are three datasets for different values of the cutoff parameter $\Lambda$, together with the $1 /(\Lambda a)$ bands. The point $a / L=0$ corresponds to the infinite volume limit.

its energy in the finite cubic volume has been calculated for various values of the box side length $L$. In order to check the consistency of our results, the calculation was carried out for several cutoff momenta $\Lambda$. For each cutoff, the three-body interaction parameterized by $H(\Lambda)$ has been adjusted such that the infinite volume binding energies are identical for all considered values of $\Lambda$. If our results are properly renormalized, the results for the different cutoffs should agree with each other up to an uncertainty of order $1 /(\Lambda a)$ stemming from the finiteness of the cutoff.

The results for the states II and III are depicted in Fig. 2 for box sizes between $L=6 a$ and $L=a / 2$. The values obtained for different cutoffs indeed agree with each other within the depicted uncertainty bands. Note that the bands do not represent corrections from higher orders of the EFT. For both states, the infinite volume limit is smoothly approached. As the volume becomes smaller, the energy of the states is more and more diminished. This corresponds to an increased binding with decreasing box size.

In the infinite volume, state III is more deeply bound than state II. Naïvely, one therefore expects the former to have a smaller spatial extent than the latter. The size of the state can be estimated via the formula $\left(-m E_{3}^{\infty}\right)^{-1 / 2}$, yielding $0.45 a$ for state II and $0.3 a$ for state III. Hence, a given finite volume should affect state II more strongly than the smaller state III. This behavior can indeed be observed. For example, considering a cubic volume with side length $L=2 a$, the energy of state II deviates $10 \%$ from the infinite volume value, whereas the corresponding difference for state III is less than one percent. On the other hand, the box size for which the energy shift of the state III amounts to $10 \%$ is roughly $1.3 a$.

In Fig. 3, the three datasets obtained for state II are plotted again, this time in comparison to the results from a calculation using the expansion of the kernel as in Ref. [38]. For large values of the box size $L$, the results of both calculations agree with each other. For volumes smaller than $1.7 a$, the result of the calculation with the expansion devi-

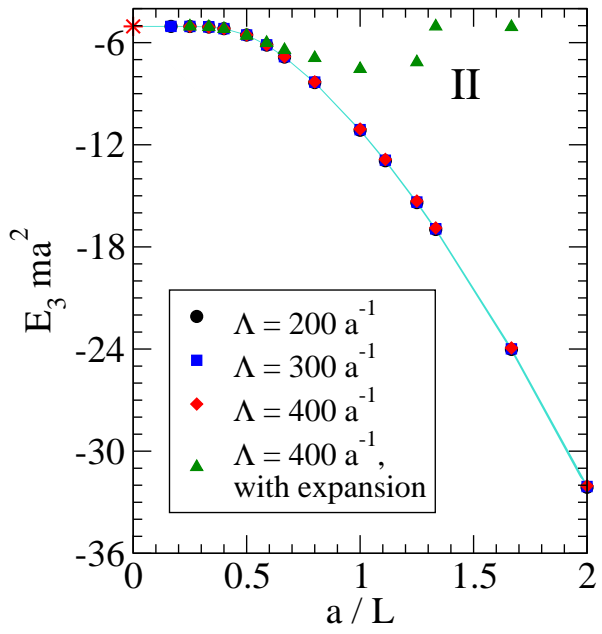

Fig. 3. Variation of the trimer energy $E_{3}$ with the side length $L$ of the cubic volume for state II. Plotted are three datasets for different values of the cutoff parameter $\Lambda$, together with the $1 /(\Lambda a)$ bands, and one dataset obtained using a Taylor expanded version of the integral kernel.

ates from the result of the full calculation. For this size of the volume, the full result differs by about $20 \%$ from the infinite volume binding energy. Accordingly, the expansion employed for the integral kernel can not be applicable any longer. In Fig. 3, results are only depicted for volumes with $L \geq a$. For smaller volumes, the results of calculations using the expansion become cutoff dependent, and are hence not are not properly renormalized anymore.

In Fig. 4, we show our results for the two states Ia and $\mathrm{Ib}$ in the same physical system characterized by $\Lambda_{*} a=$ 5.66. The volume dependence of the two states is again shown for box sizes between $L=6 a$ and $L=a / 2$. The curve corresponding to the more deeply bound state $\mathrm{Ib}$ shows a behavior that is similar to the one observed for the states II and III. The binding energy remains constant 
$19^{\text {th }}$ International IUPAP Conference on Few-Body Problems in Physics

\begin{tabular}{|c|c|c|c|c|}
\hline$L / a$ & $\Lambda a$ & $E_{3}(L) m a^{2}, \quad \ell=0,4$ & $E_{3}(L) m a^{2}, \quad \ell=0$ & $E_{2} m a^{2}$ \\
\hline \hline 1.25 & 200 & -4.30392 & -4.24545 & -3.53099 \\
\hline \multirow{3}{*}{1} & 200 & -4.57097 & -4.57753 & \multirow{3}{*}{-5.07581} \\
\cline { 2 - 5 } & 300 & -4.60298 & -4.60872 & \\
\cline { 2 - 5 } & 400 & -4.59579 & -4.60056 & -6.068 \\
\hline 0.9 & 200 & -4.31223 & -4.27927 & \multirow{2}{*}{} \\
\hline
\end{tabular}

Table 1. Energies $E_{3}(L)$ of state Ia for box sizes near $L=a$ calculated with and without admixture of the $\ell=4$ amplitude. The dimer energy $E_{2}$ is shown for comparison.

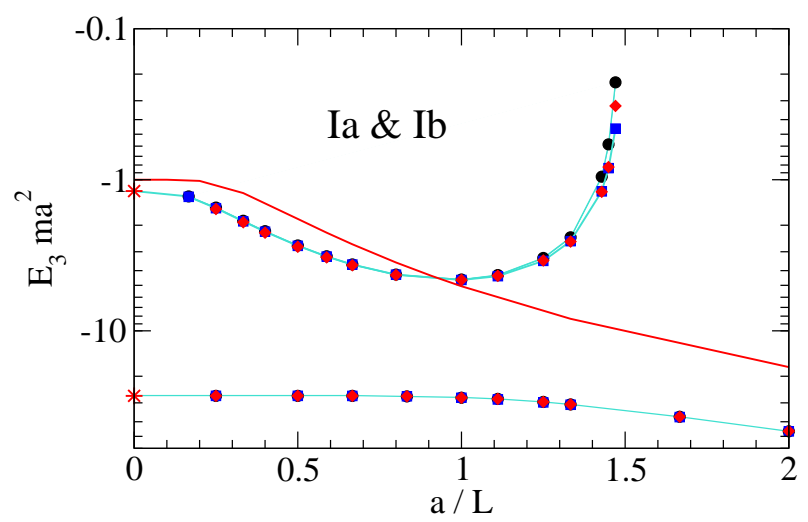

Fig. 4. Variation of the trimer energy $E_{3}$ with the side length $L$ of the cubic volume for the states Ia (upper curve) and $\mathrm{Ib}$ (lower curve). Plotted are three datasets for different values of the cutoff parameter $\Lambda$, together with the $1 /(\Lambda a)$ bands (circles: $\Lambda=200 a^{-1}$; squares: $\Lambda=300 a^{-1}$; diamonds: $\Lambda=400 a^{-1}$ ). The solid line depicts the diboson energy.

until the volume is small enough to affect the state. At this point, the energy of the state is more and more diminished as the volume becomes smaller.

The behavior of the shallow state Ia is different. In the region $L \approx a$, the binding is not further increased. The results for smaller volumes show a sharp rise of the three-body energy. The energy of the state becomes positive near $L=0.67 a$. State Ia is close to the threshold for boson-diboson scattering in the infinite volume located at $E_{D}=-1 /\left(m a^{2}\right)$. For comparison, we calculated the energy of the physical diboson according to [24]. The resulting curve is the solid line in Fig. 4. Like the energy of the threebody bound states, the energy of the diboson is diminished in finite volumes. For volumes of the size $L \approx 1.2 a$, the energy of the three-body state Ia becomes larger than the diboson energy and starts to grow. This behavior is consistent with the observation that states are always shifted away from the threshold in a finite volume. In the two-body sector, for example, continuum states have been shown to have a power law dependence on the volume, while the volume dependence of bound states is dominated by exponentials [24]. The data shown for state Ia can be explained by an exponential for $L>a$, which characterizes the state as a bound state. For $L<a$, the data is consistent with a power law, indicating the state indeed behaves like a boson-diboson scattering state if its energy is above the diboson energy.
The other investigated states do not show such a transition since their energy is well below the diboson energy for all considered volumes. It is unclear whether other states would show a similar behavior for smaller box sizes. If this is not the case, the transition from bound to unbound would occur only for states with infinite volume binding energies up to a critical value. For a conclusive analysis of the nature of the described transition, more data is still needed. It would be interesting to see whether such a transition shows up in lattice data for a state that is very close to the diboson threshold in the infinite volume.

Since the size of the finite volumes where the state crosses the diboson energy is comparable to the size of the state itself, the breaking of the spherical symmetry may be a relevant effect here. To assess the influence of the higher partial waves, we extract from Eq. (6) two coupled equations for the s-wave amplitude $F_{0}(\ell=0)$ and the $\ell=4$ amplitude $F_{4}$. These coupled equations are then solved in a coupled channel approach. The energies of state Ia obtained by this method are summarized and compared to the s-wave only results in Table 1 . For $L=1.25 a$, the state is still below the dimer state. The inclusion of the higher partial wave leads to a small downward shift in the energy. For $L=a$, we have done calculations using 3 different cutoffs. The results for different cutoffs agree to two significant digits indicating the results are properly renormalized, but the binding is slightly reduced by the $\ell=4$ contribution. For $L=0.9 a$, the effect of the higher partial wave is again a small downward shift. All results show only a deviation of about $1 \%$ from the s-wave only result. In summary, we find that the correction from the $\ell=4$ admixture is extremely small. Moreover, the correction is of the same order of magnitude as the finite cutoff corrections in our calculation and a more quantitative statement requires improved numerical methods.

In order to establish an estimate of typical corrections from higher partial waves, we have also performed calculations including the $\ell=4$ contributions for the more deeplybound state II. The resulting numbers are given in Tab. 2 . The investigated box sizes are about three times larger than the state itself. The contribution of the higher partial wave is now several percent. This is still a small correction but considerably larger than the finite cutoff uncertainty. This suggests that the extremely small corrections for state Ia are related to its unusual behavior. The dominance of the s-wave my be associated with the closeness of the state to the threshold. A more detailed investigation of higher partial waves including a description of the numerical methods will be the subject of a future publication. 
EPJ Web of Conferences

\begin{tabular}{|c|c|c|c|}
\hline$L / a$ & $\Lambda a$ & $E_{3}(L) m a^{2}, \quad \ell=0,4$ & $E_{3}(L) m a^{2}, \quad \ell=0$ \\
\hline \hline \multirow{3}{*}{1} & 200 & -11.86 & -11.15 \\
\cline { 2 - 4 } & 400 & -11.79 & -11.08 \\
\hline \multirow{2}{*}{0.7} & 200 & -19.06 & -20.70 \\
\cline { 2 - 4 } & 400 & -18.97 & -20.64 \\
\hline
\end{tabular}

Table 2. Energies $E_{3}(L)$ of state II for box sizes $L=a$ and $L=0.7 a$ calculated with and without admixture of the $\ell=4$ amplitude.

Since the shallower state Ia is more affected by a given finite volume than the deeper state $\mathrm{Ib}$, the ratio of the energies of the states is changing. In the infinite volume, this ratio is 23.08 . For $L=1.5 a$, just before the shallow state crosses the dimer energy, this ratio has decreased to 7.4. Note that this ratio differs from the discrete scaling factor $\exp \left(2 \pi / s_{0}\right) \approx 515$ even in the infinite volume limit. This behavior is expected for shallow states close to the bound state threshold [1]. The ratio $\exp \left(2 \pi / s_{0}\right)$ will be approached when deeper states are considered. For example, the infinite volume ratio of state $\mathrm{Ib}$ and the much more deeply bound state Ic is 344 and already closer to the discrete scaling factor 515 .

\subsection{Negative scattering length}

Now we turn to systems with $a<0$. In this regime, the two-body interaction is attractive but no diboson bound state exists in the infinite volume limit. The only possible breakup process for a three-boson bound state is therefore the breakup into three single bosons. The threshold for this process is $E=0$. As for the case with positive scattering length, we choose states with different energies in the infinite volume:

$$
\begin{array}{rll}
\text { NI: } & E_{3}^{\infty}=-9 /\left(m a^{2}\right), & \Lambda_{*} a=5.55, \\
\text { NII: } & E_{3}^{\infty}=-4 /\left(m a^{2}\right), & \Lambda_{*} a=4.23, \\
\text { NIII: } & E_{3}^{\infty}=-0.2 /\left(m a^{2}\right), & \Lambda_{*} a=2.60 .
\end{array}
$$

Here, $E_{3}^{\infty}$ is the trimer energy in the infinite volume. For each of these states, its energy in the finite cubic volume has been calculated for various values of the box size $L$. In order to check the consistency of our results, the calculation was carried out for several cutoff momenta $\Lambda$. For each cutoff, the three-body interaction parameterized by $H(\Lambda)$ has been adjusted such that the infinite volume binding energies are identical for all considered values of $\Lambda$.

The results for the states NI and NII are shown in Fig. 6 for box sizes between $L=2 a$ and $L=a / 2$. The results for the state NIII with box sizes between $L=8 a$ and $L=a / 2$ are depicted in Fig. 5. The values obtained with different cutoffs all agree with each other within the uncertainty bands indicating proper renormalization. Our findings are similar to those in the positive scattering length regime. All three states smoothly approach the infinite volume limit. As the box size becomes smaller the energy of the state is more and more diminished. The overall behavior is identical to the one in the positive scattering length case described in the previous section.

The more deeply bound a state is in the infinite volume, the smaller is its spatial extent. Estimating the size via the formula $\left(-m E_{3}^{\infty}\right)^{-1 / 2}$ yields $a / 3$ for state NI, $a / 2$

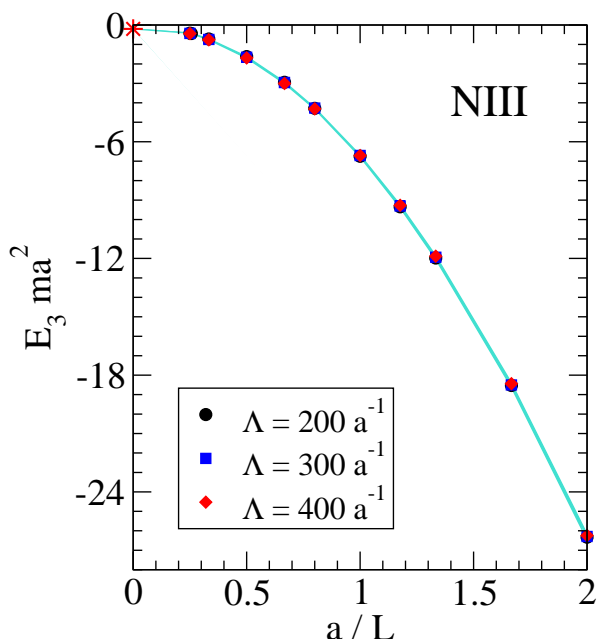

Fig. 5. Variation of the trimer energy $E_{3}$ with the side length $L$ of the cubic volume for the states NIII. Plotted are three datasets for different values of the cutoff parameter $\Lambda$, together with the $1 /(\Lambda a)$ bands.

\begin{tabular}{|l||c|c|c|}
\hline State & NI & NII & NIII \\
\hline \hline$E_{3}^{\infty} m a^{2}$ & -9 & -4 & -0.2 \\
$E_{3}(L=a) m a^{2}$ & -11.6 & -8.7 & -6.7 \\
$\delta_{\text {rel }}$ & $29 \%$ & $118 \%$ & $3250 \%$ \\
$L_{10 \%} / a$ & 1.2 & 1.8 & 7.2 \\
$L_{100 \%} / a$ & 0.7 & 1.05 & 4.2 \\
\hline
\end{tabular}

Table 3. For the states NI, NII and NIII, the infinite volume energy $E_{3}^{\infty}$ and the energy in a finite volume with side length $L=a$ are shown together with the relative deviation from the infinite volume value $\delta_{\text {rel }}=\left(E_{3}-E_{3}^{\infty}\right) / E_{3}^{\infty}$. Also given are the box sizes leading to an energy shift of $10 \%$ and $100 \%$, respectively.

for state NII, and $2.2 a$ for state NIII. A given finite volume should therefore affect state NII more than state NI, and state NIII should be the most affected. When considering a cubic volume with side length $L=a$, we find the energies given in Table 3 . The relative deviation of state NI is indeed four times smaller than the shift for state NII and a hundred times smaller than the shift for the shallow state NIII. On the other hand, the box length $L_{10 \%}$, for which the energy of each states deviates $10 \%$ from its infinite volume value, is smaller the more deeply bound a state is. These values are also given in Table 3.

\section{Conlusions}

We have extended our earlier studies of the Efimov spectrum in a cubic box with periodic boundary conditions [38]. 

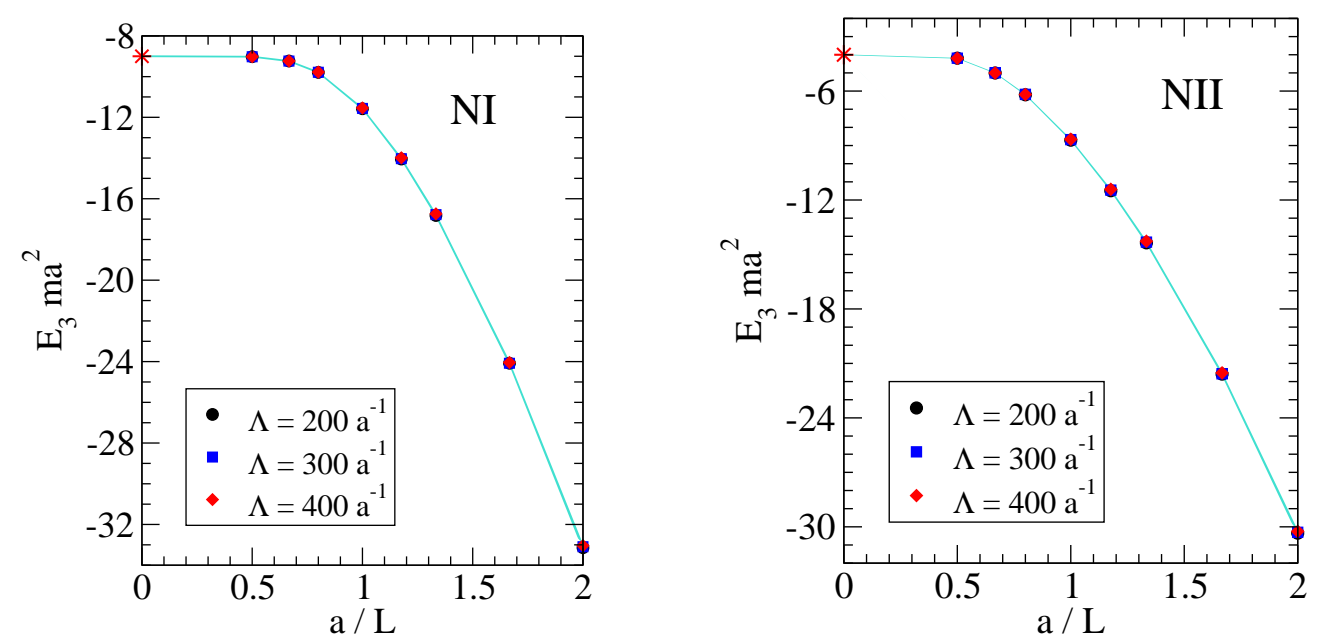

Fig. 6. Variation of the trimer energy $E_{3}$ with the side length $L$ of the cubic volume for the states NI (left) and NII (right). Plotted are three datasets for different values of the cutoff parameter $\Lambda$, together with the $1 /(\Lambda a)$ bands. The point $a / L=0$ corresponds to the infinite volume limit.

The knowledge of the finite volume modifications of the spectrum is important in order to understand results from future 3-body lattice calculations. Using the framework of EFT, we have derived a general set of coupled sum equations for the Efimov spectrum in a finite volume.

Specializing to $\ell=0$, we have calculated the spectrum for both positive and negative scattering lengths and verified the renormalization in the finite box explicitly. We have removed the expansion for small finite volume shifts used in Ref. [38] and presented an extension that can be applied to arbitrarily large shifts. Typically, the binding of all three-body states increases as the box size is reduced. Moreover, we provided a more detailed discussion of the technical details and our numerical method. We have investigated the breakdown of the linear approximation in detail and find that the expansion is applicable as long as the finite volume shift in the energy is not larger than 15$20 \%$ of the infinite volume energy.

For positive scattering lengths, we have investigated a spectrum of three states in the same physical system and studied the behavior of the shallowest state in the vicinity of the dimer energy which specifies the scattering threshold in the infinite volume. We found that this state drastically changes its behavior as a function of the box length once its energy becomes equal to the dimer energy. At this point the energy of the shallowest three-body state starts to grow and eventually becomes positive. The observed behavior is consistent with exponential suppression of the finite volume corrections below the dimer energy and power law suppression above.

The effect of an admixture of the $\ell=4$ partial wave has been investigated for two different three-body states for box sizes of the order of the size of the state. For a generic state well separated from threshold, we found corrections of the order of a few percent for volumes that are about three times larger than the state itself. For the state closest to threshold, the effect of this admixture turns out to be surprisingly small for volumes about twice as large as the state and was found to be less than $1 \%$. As the finite cutoff corrections for this state are about the same size, a more quantitative study requires an improved treatment of these corrections. Such studies are in progress.

Finally, our method should be extended to the threenucleon system. This requires also the inclusion of higher order corrections in the EFT and finite temperature effects as lattice calculations will inevitably be performed at a small, but non-zero, temperature. Work in these directions is in progress. With high statistics lattice QCD simulations of three-baryon systems within reach [22], the calculation of the structure and reactions of light nuclei appears now feasible in the intermediate future. Our results demonstrate that the finite volume corrections for such simulations are calculable and under control. This also opens the possibility to test the conjecture of an infrared limit cycle in QCD for quark masses slightly larger than the physical values [18].

\section{References}

1. E. Braaten and H.-W. Hammer, Phys. Rept. 428, 259 (2006) [arXiv:cond-mat/0410417].

2. L. Platter, Few Body Syst. 46, 139 (2009) [arXiv:0904.2227 [nucl-th]].

3. V. Efimov, Phy. Lett. 33B, 563 (1970).

4. V. Efimov, Sov. J. Nucl. Phys. 29, 546 (1979).

5. P.F. Bedaque, H.-W. Hammer, and U. van Kolck, Phys. Rev. Lett. 82, 463 (1999) [arXiv:nuclth/9809025]; Nucl. Phys. A 646, 444 (1999) [arXiv:nucl-th/9811046].

6. T. Kraemer, M. Mark, P. Waldburger, J.G. Danzl, C. Chin, B. Engeser, A.D. Lange, K. Pilch, A. Jaakkola, H.-C. Nägerl, and R. Grimm, Nature 440, 315 (2006).

7. S. Knoop, F. Ferlaino, M. Mark, M. Berninger, H. Schoebel, H.-C. Naegerl, R. Grimm Nature Physics 5, 227 (2009) [arXiv:0807.3306 [cond-mat]]. 
8. T. B. Ottenstein, T. Lompe, M. Kohnen, A. N. Wenz, S. Jochim, Phys. Rev. Lett. 101, 203202 (2008) [arXiv:0806.0587 [cond-mat]].

9. J. H. Huckans, J. R. Williams, E. L. Hazlett, R. W. Stites, K. M. O’Hara, Phys. Rev. Lett. 102, 165302 (2009) [arXiv:0810.3288 [physics.atom-ph]].

10. G. Barontini, C. Weber, F. Rabatti, J. Catani, G. Thalhammer, M. Inguscio, F. Minardi, Phys. Rev. Lett. 103, 043201 (2009) [arXiv:0901.4584v1 [condmat.other]].

11. N. Gross, Z. Shotan, S. Kokkelmans, L. Khaykovich, Phys. Rev. Lett. 103, 163202 (2009) [arXiv:0906.4731v1 [cond-mat.other]].

12. M. Zaccanti, B. Deissler, C. D'Errico, M. Fattori, M. Jona-Lasinio, S. Müller, G. Roati, M. Inguscio, G. Modugno, Nature Physics 5, 586 (2009) [arXiv:0904.4453v1 [cond-mat.quant-gas]].

13. D. L. Canham and H.-W. Hammer, Eur. Phys. J. A 37, 367 (2008) [arXiv:0807.3258 [nucl-th]] and references therein.

14. K.G. Wilson, Nucl. Phys. Proc. Suppl. 140, 3 (2005) [arXiv:hep-lat/0412043].

15. S.R. Beane, P.F. Bedaque, M.J. Savage, and U. van Kolck, Nucl. Phys. A 700, 377 (2002) [arXiv:nuclth/0104030].

16. S.R. Beane and M.J. Savage, Nucl. Phys. A 717, 91 (2003) [arXiv:nucl-th/0208021]; Nucl. Phys. A 713, 148 (2003) [arXiv:nucl-th/0206113].

17. E. Epelbaum, U.-G. Meißner, and W. Glöckle, Nucl. Phys. A 714, 535 (2003) [arXiv:nucl-th/0207089].

18. E. Braaten and H.-W. Hammer, Phys. Rev. Lett. 91, 102002 (2003) [arXiv:nucl-th/0303038].

19. E. Epelbaum, H.-W. Hammer, U.-G. Meißner and A. Nogga, Eur. Phys. J. C 48, 169 (2006) [arXiv:hep$\mathrm{ph} / 0602225]$.

20. H.-W. Hammer, D.R. Phillips and L. Platter, Eur. Phys. J. A 32, 335 (2007) [arXiv:0704.3726 [nucl-th]].

21. S. R. Beane, P. F. Bedaque, K. Orginos and M. J. Savage, Phys. Rev. Lett. 97, 012001 (2006) [arXiv:heplat/0602010].

22. S. R. Beane et al., Phys. Rev. D 80 (2009) 074501 arXiv:0905.0466 [hep-lat].

23. S. R. Beane, K. Orginos and M. J. Savage, Int. J. Mod. Phys. E 17, 1157 (2008) [arXiv:0805.4629 [hep-lat]].

24. S. R. Beane, P. F. Bedaque, A. Parreno and M. J. Savage, Phys. Lett. B 585, 106 (2004) [arXiv:heplat/0312004].

25. S. Tan, Phys. Rev. A 78, $013636 \quad$ (2008) [arXiv:0709.2530 [cond-mat]].

26. S. R. Beane, W. Detmold and M. J. Savage, Phys. Rev. D 76, 074507 (2007) [arXiv:0707.1670 [hep-lat]].

27. W. Detmold and M. J. Savage, Phys. Rev. D 77, 057502 (2008) [arXiv:0801.0763 [hep-lat]].

28. S. R. Beane, W. Detmold, T. C. Luu, K. Orginos, M. J. Savage and A. Torok, Phys. Rev. Lett. 100 (2008) 082004 [arXiv:0710.1827 [hep-lat]].

29. W. Detmold, M. J. Savage, A. Torok, S. R. Beane, T. C. Luu, K. Orginos and A. Parreno, Phys. Rev. D 78, 014507 (2008) [arXiv:0803.2728 [hep-lat]].
30. W. Detmold, K. Orginos, M. J. Savage and A. Walker-Loud, Phys. Rev. D 78, 054514 (2008) [arXiv:0807.1856 [hep-lat]].

31. L. Pricoupenko and Y. Castin, J. Phys. A 40, 12863 (2007) [arXiv:0705.1502 [cond-mat]].

32. M. Lüscher, Nucl. Phys. B 354, 531 (1991).

33. M. Lüscher, Nucl. Phys. B 364, 237 (1991).

34. V. Bernard, U.-G. Meißner and A. Rusetsky, Nucl. Phys. B 788, 1 (2008) [arXiv:hep-lat/0702012].

35. V. Bernard, M. Lage, U.-G. Meißner and A. Rusetsky, JHEP 08, 024 (2008) [arXiv:0806.4495 [hep-lat]].

36. F. C. von der Lage and H. A. Bethe, Phys. Rev. 71, 612 (1947).

37. S. L. Altmann and A. P. Cracknell, Rev. Mod. Phys. 37, 19 (1965).

38. S. Kreuzer and H.-W. Hammer, Phys. Lett. B 673, 260 (2009) [arXiv:0811.0159 [nucl-th]]. 